\title{
Decision making and the P300 component of the cortical evoked response*
}

\author{
JOHN W. ROHRBAUGH $\dagger$, EMANUEL DONCHIN, and CHARLES W. ERIKSEN \\ University of Illinois, Champaign/Urbana, Illinois 61820
}

\begin{abstract}
The amplitude of P300, and of other components of the evoked potential, was examined during a task which required $S s$ to make a response appropriate to the conjoint properties of two rapidly successive visual patterns. In one set of conditions, the task was structured such that both stimuli were needed for the choice judgment. In another set of conditions, the task was changed from choice to simple RT by presenting the patterns in a predictable order. It was observed that P300 was enhanced during the choice R'T conditions and that this enhancement was present only for the P300 following the stimulus permitting the choice and not the other, relevant but not decisive, stimulus appearing in close temporal proximity. An appreciable degree of independence between $\mathrm{P300}$ and other components was indicated by the appearance of P300 under conditions in which other evoked potential components were entirely refractory. The data support an account for P300 in terms of poststimulus processes, such as decision making, and not in terms of preparatory adjustments.
\end{abstract}

Since the initial report by Sutton et al (1965), a considerable literature has dealt with a late positive component (with a latency of about $300 \mathrm{msec}$ and labeled P300 in the present report) of the human average evoked potential (AEP). Without exception, these reports suggest that the amplitude of $\mathrm{P} 300$ varies according to the role which the eliciting stimulus plays in the S's task. It has been shown, for example, that P300 is enhanced when the stimulus "resolves uncertainty" (Sutton et al, 1965, 1967; Tueting et al, 1971), when it is made "task relevant" (Donchin \& Cohen, 1967), and when it is novel or unexpected (Ritter et al, 1968).

Although there seems to be little doubt that P300 can be elicited, or enhanced, by a class of variables that are often labeled "psychological" (as distinct from the physical parameters of stimulation), there is little consensus concerning the nature of the critical psychological variables. In fact, the diversity of situations in which P300 can be enhanced may indicate that P300 has no single psychological concomitant. The variety of hypotheses put forward to account for the phenomenon may be divided into two general classes of explanation. One class of explanation asserts that differences in $\mathrm{P} 300$ reflect differential states of preparedness, either specific or nonspecific, which develop prior to significant stimuli and influence the registration or analysis of these stimuli (Karlin, 1970;

*This investigation was supported by United States Public Health Service Research Grant MH-01206 and the United States Public Health Service Career Program Award K6-MH-22014 to C.W.E.; by Grant NSF GB-30999 from the National Science Foundation to E.D.; and by Grant OEG-5-70-0030 (508) from the US. Office of Education, also to E.D. This report is based on portions of a dissertation submitted by J,W.R. in partial fulfillment of the requirements for the PhD dexee in Psychology at the University of Illinois. This study was presented in a preliminary format before the annual convention of the American EEG Society. Houston, 1972.

tR eprint requests should be addressed to John W. Rohrbaugh whose present address is: University of California, Department of Psychology, Los Angeles, California 90024.
Natanen, 1970). Accordingly, the P300 for any stimulus presented during an attentive state will be enhanced, in the same manner as earlier AEP components are enhanced by arousal or attention (see Eason et al, 1969).

Contrasted with these "tonic" accounts for P300 are accounts which view $\mathrm{P} 300$ as reflecting a more "phasic" process arising after, and in response to, significant stimuli. While it has been suggested that this phasic process may be an abrupt reactive dissipation of the preparatory state upon task completion (Karlin, 1970), there are conflicting data (summarized by Tueting \& Sutton, 1973) indicating some degree of independence between P300 and the electrocortical signs of preparation. Another possibility is that $\mathrm{P} 300$ reflects the cognitive responses demanded by the S's task, since P300-eliciting stimuli normally call for an appreciable measure of cortical information processing. Donchin et al (1973) have speculated that a single indispensable cognitive process (an "intracortical processor"), invoked by otherwise dissimilar analysis programs, is responsible for the appearance of P300. A likely candidate for this indispensable cognitive process is the process of decision making, as proposed by Smith et al (1970).

The "phasic" view of $\mathrm{P} 300$ is consistent with reports that differentially enhanced P300s can be obtained under conditions which preclude differential adjustments in arousal (Ritter et al, 1968; Donchin et al, 1972). Furthermore, selectively enhanced $P 300$ s have been elicited by relevant stimuli even when the physical similarity of relevant and irrelevant stimuli makes it implausible that any selective filtering or intensification could transpire (Donchin \& Cohen, 1967; Harter \& Salmon, 1972; Hillyard et al, 1973). The phasic interpretation gains credence also from the fact that the amplitude and form of P300 seem to be independent of the modality, or even the presence of the eliciting stimuli. It has been demonstrated, for example, that 

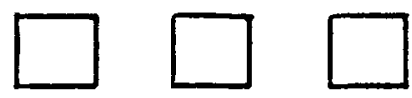

a
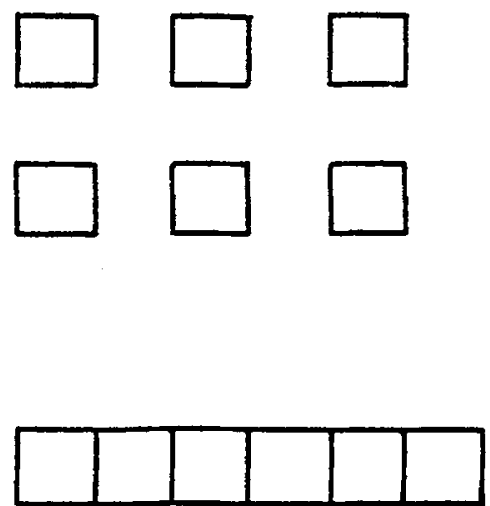

b
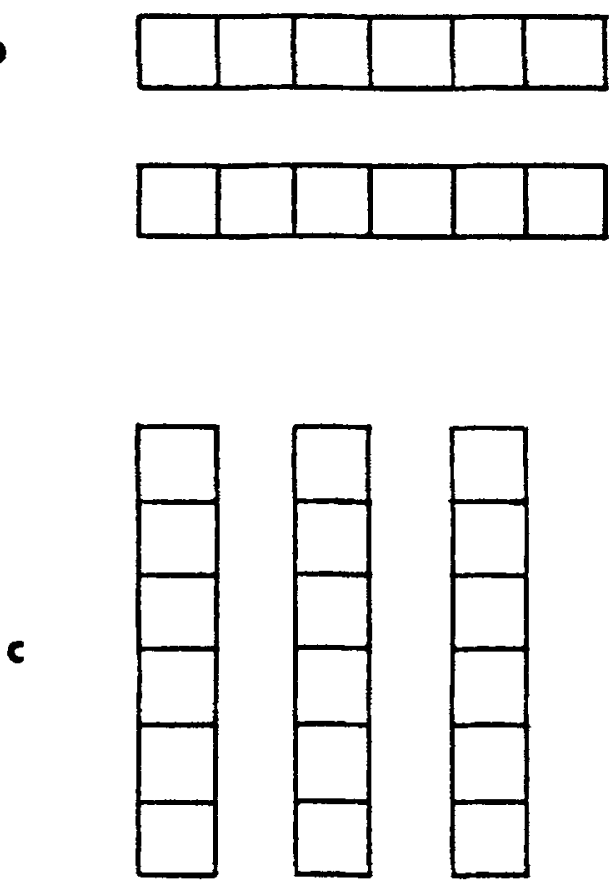

Fig. 1. Two identical grids of illuminated squares, each as outlined in (a), can be intercalated to yield either horizontal stripes (b) or vertical stripes (c).

P300 appears even to the omission of an expected stimulus from a stimulus train (Klinke et al, 1968; Picton et al, in press; Sutton et al, 1967).

We report here a study which lends further support to the phasic interpretation of $\mathrm{P} 300$. Our data demonstrate that the enhancement of $\mathrm{P} 300$ arises from a poststimulus process, such as decision making, and not directly or invariably because of preparatory adjustments in arousal or attention. Our Ss had to make a judgment appropriate to the conjoint properties of two rapidly successive visual stimuli, both of which were presented during comparable states of preparation. The two stimuli were presented in such close temporal succession that changes in tonic preparedness between the two were unlikely. Moreover, both stimuli were relevant and were spatially interlocking and contiguous, so as to discourage any sort of selective tuning. It is a crucial feature of our design that, while both stimuli were relevant, only the second of the two stimuli allowed Ss to make the required judgment. By comparing the AEPs elicited by the two stimuli under a variety of experimental conditions, we could assess the degree to which P300 amplitude is determined by tonic variables, and could speculate as to the nature of the phasic variables which affect P 300 .

\section{METHOD}

We used a task initially developed by Rohrbaugh and Eriksen (in preparation) for studying the temporal integration of two visual stimuli. Figure 1 depicts the stimuli devised for these experiments. Two identical grids of illuminated squares ( $\mathrm{Sl}$ and $\mathrm{S2}$, each as outlined in Fig. 1 a) could be intercalated to yield either horizontal stripes (Fig. 1b) or vertical stripes (Fig. 1c). Temporal integration was examined by introducing an interval (stimulus onset asynchrony, or SOA) between brief exposures of the two grids.

The task was structured in such a manner that both S1 and S2 were needed to determine the orientation of the composite stripe. While S1 provided crucial information, the choice decision was necessarily delayed until the onset of S2. Rohrbaugh and Eriksen reasoned that the decision latency following $\mathrm{S} 2$ (as measured by choice reaction times) would depend upon the manner in which the two grids were integrated. If the SOA were so short that the iconic persistence from $S 1$ was still viable when $S 2$ appeared, the two grids could be visually integrated into a composite stripe similar to that when both had been presented simultanenusly. The orientation could not be determined so readily at SOAs longer than the persistence from $\mathrm{S} 1$, since this decision would involve integration of S2 with abstracted spatial information about $S 1$, retrieved from some more permanent memory. Response latencies varied in a manner consistent with these expectations: reaction time (RT) following S2 was nearly $60 \mathrm{msec}$ longer at SOAs exceeding $200 \mathrm{msec}$ than at $\mathrm{SOA}=0 \mathrm{msec}$.

If $P 300$ is a concomitant of decision making, an enhanced P300 should be elicited by S2 only and should appear at an appropriate latency after S2, even though both S1 and S2 are highly task relevant and occur within temporal intervals so brief that attentional shifts between them are unlikely. We were able to emphasize the dependence of P300 enhancement on S's decisions by presenting stimuli in two series, with different sequential restraints. In the random (R) series the stripe orientation on each trial was randomly assigned, with a constraint that each orientation occur with a probability of 0.5 . In the alternating (A) series, Ss were informed what the orientation of the first stimulus would be, and thereafter the orientation alternated throughout the block of trials. Ss were instructed to withhold response until the onset of S2, effectively simplifying the A series task from choice to simple RT. It was expected that P300 would be attenuated or absent in the A series, even though physically identical stimuli and motor requirements were employed in the two series (see Sutton et al, 1965).

\section{Subjects}

Four male University of lllinois students were paid for participating in the experiment. All were right-handed and had normal or corrected to normal vision. A fifth $S$ was excluded because he was unable to maintain a stable fixation. All Ss had 
extensive practice in similar experiments, and were each given an additional $2 \mathrm{~h}$ practice in the tasks described below.

\section{Apparatus}

Stimuli were exposed in a specially constructed tachistoscope. Individual squares in the stimulus grid were highly polished $1 / 2 \mathrm{x}$ $1 / 2 \times 2 \frac{1}{2}$ in. blocks of transparent Plexiglas, with the $1 / 2$-in.-sq surface facing the viewer capped with $1 / 8$-in. white Plexiglas to ensure homogeneous light diffusion across the entire surface. Eighty-one such blocks were clamped together into a 9 by 9 matrix with adjacent squares in the matrix separated by thin sheets of silvered mylar to prevent light leakage and to increase internal reflection.

Each square was supplied white light by one of 10 Sylvania F4T5/CWX fluorescent lamps. The lamps were controlled by Iconix timing equipment and other circuitry which has been described in detail elsewhere (Eriksen et al, 1969). Light was transmitted to the appropriate square by individual 18-in. lengths of $1 / 8$-in. plastic fiber-opties. An aluminum ferrule was pressed over the source end of each fiber-optic and was then inserted into a hole bored in a metal block mounted on the lamp housing. The luminance of each square could be separately adjusted by varying the depth of insertion. The ferrules were inserted so that all squares were equated to a luminance of $1.0 \mathrm{fL}$ (as measured with a Spectra UB $1 / 2$ Spot Photometer) and locked in place with set screws. The background had no measurable luminance. A fixation cross was fashioned by masking the face of an additional square and reflecting the image from a thin sheet of transparent vinyl located so as to place the cross in the same apparent plane as the stimulus matrix. The cross was dimly illuminated by an incandescent lamp.

The entire display is schematized in Fig. 2. The hatched portions of the display either were masked or never were illuminated. All squares labeled with the same number received light from a common source. The narrow outline border surrounding the display defined the perimeter of the viewing field and was used variously to provide a spatial referent and to mark the beginning of the foreperiod. Stripes were fabricated from those sets of squares illuminated by Lamps 1, 2, 3, and 4 . If, for example, $\mathrm{Sl}$ were those squares illuminated by Lamp 1 and $S 2$ were those squares illuminated by Lamp 2, the composite stripe would be oriented horizontally.

Ss were comfortably seated in an automobile bucket seat placed in an electrically shielded and sound-deadened booth. Ss were instructed to rest their heads gently against a viewing hood, from which they monocularly viewed the display at the end of a 92-in. horizontal alley. At this distance, each individual square subtended $19 \times 19$ min visual angle, and each composite stripe was $1 \mathrm{deg} 52 \mathrm{~min}$ long. A field stop located $22 \mathrm{in}$. from the right eye occluded all but a 2 -deg 50 -min square field.

The response console contained a $2 \frac{1}{2} 2$-in. vertical lever which Ss moved either to the left or to the right to indicate the appropriate stripe orientation. Very little pressure was required to actuate the lever, therebclosing a microswitch and placing a low-voltage pulse on the recording tape. A weak but clearly audible click was emitted by switch closure.

\section{Procedures}

S1 and S2 were presented at SOAs of $0,15,30,60,90,120$, 240 , and $480 \mathrm{msec}$. The exposure durations of $S 1$ were also varied to provide a check on our interpretation in terms of temporal integration. In the intermittent (I) conditions, both $\mathrm{S} 1$ and S2 were exposed only for $30 \mathrm{msec}$ with a dark interval between them (provided the SOA exceeded the 30-msec exposure duration of $S 1$ ). In the continuous (C) conditions, S1 appeared at the appropriate $S O A$ before $S 2$, but remained on continuously until the termination of $S 2$. As in the I condition, S2 was exposed only for $30 \mathrm{msec}$. We reasoned that the $\mathrm{C}$ condition would permit visual integration over all SOAs, so that the decision latency following $S 2$ onset would remain fairly constant as the SOA progressed from 0 to $480 \mathrm{msec}$. All of these

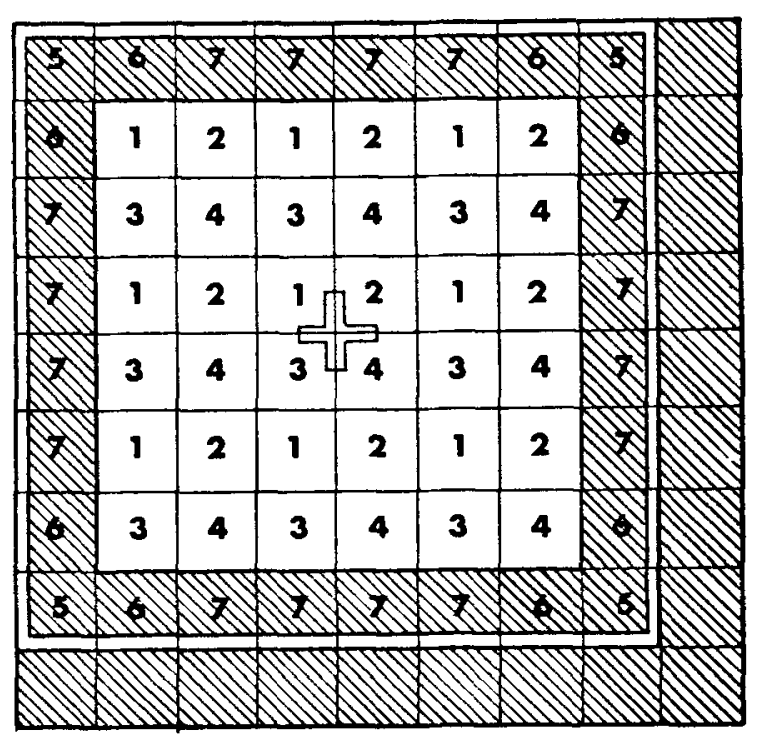

Fig. 2. Schematized viewing surface of the tachistoscope.

stimulus combinations were given under the $R$ and $A$ series, yielding 32 different experimental conditions.

Referring again to $F$ ig. 2 , there are eight permutations of those stimuli illuminated by Lamps $1,2,3$, and 4 which yield stripes. Four of these, 1-2, 2-1,3-4, and 4-3, are horizontal, and the remaining four, $1-3,3-1,2-4$, and $4-2$, are vertical. If, as in the present experiment, these $\mathrm{S} 1-\mathrm{S} 2$ pairs are presented with equal frequency, then one member of the pair by itself does not provide information sufficient to determine the orientation of the completed stripe. The grid illuminated by Lamp 1, for example, is used equally often to form both horizontal and vertical stripes. Each of the above S1-S2 pairs occurred four times during a block of 32 trials. For the $\mathbf{R}$ series, these blocks followed 16 random orders and their reverses. An equal number of orders were generated with the additional restraints imposed in the A series. Each $S$ participated in eight experimental sessions, each session under a single SOA. The order of SOA presentation was balanced across $\mathrm{Ss}$ as fully as possible. Within a session, the first four blocks of trials contained each of the four $\mathrm{I} / \mathrm{C}$ by $\mathrm{R} / \mathrm{A}$ combinations, and the four combinations were presented in reverse order during the second half. Throughout the experiment, then, each $\mathrm{S}$ encountered 64 trials under each of the 32 SOA by $/ / C$ by R/A conditions.

The intertrial field was dark except for the fixation cross and the corners of the outline border illuminated by Lamp 5 . Upon initiation of the trial by $E$, the remainder of the border (illuminated by Lamps 6 and 7) also appeared. S2 always appeared $3,000 \mathrm{msec}$ following this stimulus and was preceded by $\mathrm{S} 1$ at the appropriate SOA. The field did not revert to the intertrial configuration until an interval of $6,000 \mathrm{msec}$ had elapsed, and Ss were cautioned against making any unnecessary eye or body movements throughout this interval. Aside from the previously mentioned click emitted by the response console, the equipment produced no audible transient noises during the stimulation sequence.

After cleaning the scalp with acetone, chlorided Grass silver cup electrodes were applied with Grass electrode paste at a resistance of $5,000 \mathrm{ohms}$ or less. EEG was recorded from the vertex and right occiput $(\mathrm{Cz}$ and 02 in the International 10-20 system) referred to linked earlobes. Two additional electrodes were applied to the left temple and inferior orbit to monitor eye movements, and Ss were grounded with a seventh electrode placed on the forehead.

The EEG was amplified with Grass Model 7P5A wideband ac preamplifiers and associated driver amplifiers, set to a half-amplitude bandpass of 0.3 to $35 \mathrm{~Hz}$ and calibrated to a gain 

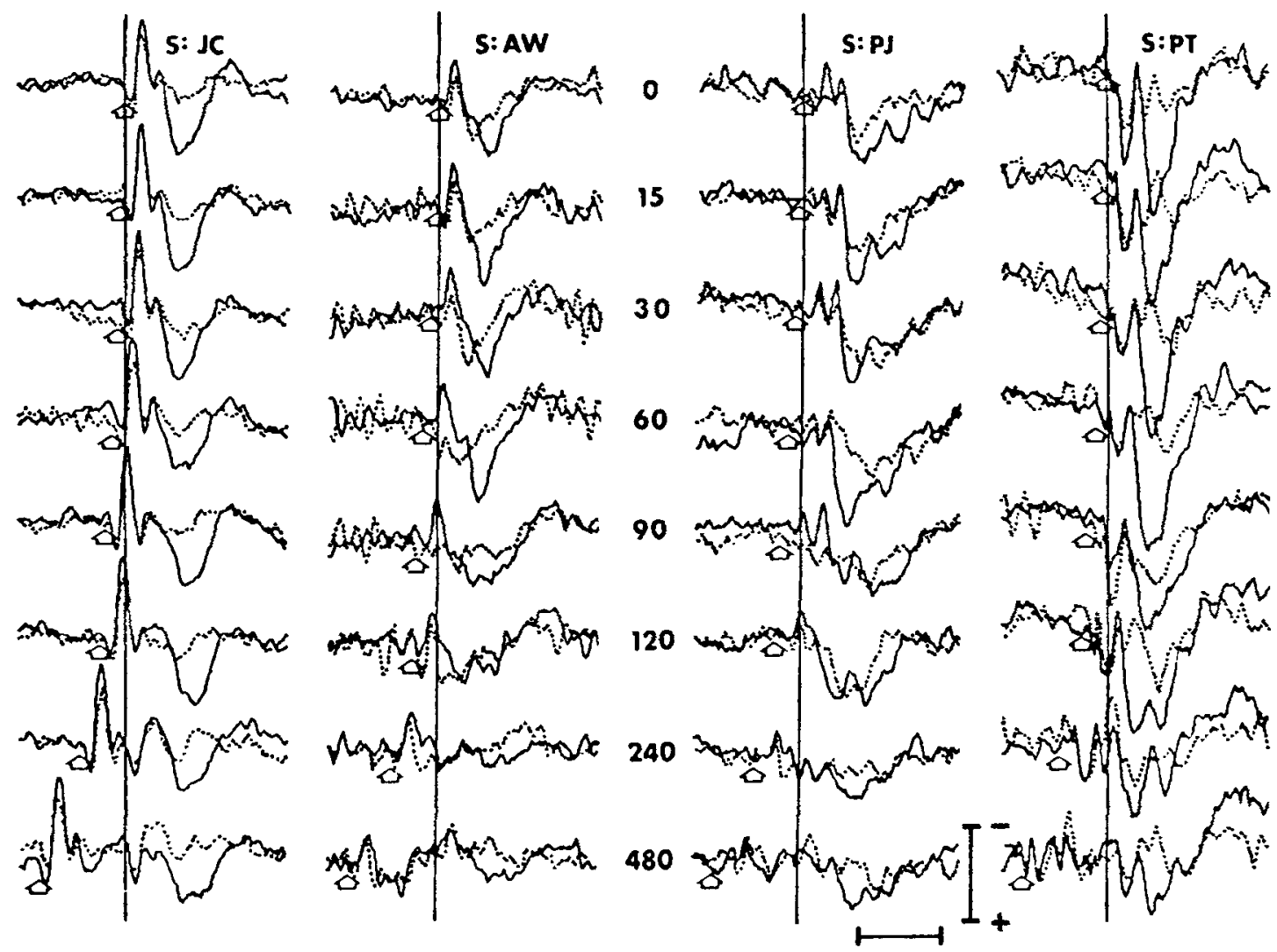

Fig. 3. AEPs for the RI (solid traces) and AI (dotted traces) conditions. The onset of S1 is indicated by the outline arrow and $S 2$ by the vertical line. Each column is from a different $S$, and each row under the SOA indicated in the center of the figure. The two left columns were recorded from the occiput and those on the right from the vertex. Vertical calibration: 10 microV. Horizontal calibration: $500 \mathrm{msec}$. Positivity is indicated by a downward deflection.

of $10^{3}$. EOGs were amplified by a modified Grass Model SPlA dc amplifier. All data (including pulses indicating S's responses and pulses synchronous with the stimulation sequence) were recorded on a Mnemotron Model 700 1/2-in. FM tape recorder. EEG and EOG channels were digitized and averaged using an IBM 1800 computer. EOG and the vertex channels were monitored continuously on an oscilloscope during data acquisition. Averaged EOGs showed no systematic fluctuations comparable to those observed at scalp sites.

Following application of the electrodes, Ss dark adapted for $7 \mathrm{~min}$ and received 32 practice trials under the $R I$ condition at the current SOA. Experimental trials proceeded at the rate of about 1/10 sec. Each block of trials was preceded by 4 practice trials to acquaint $S s$ with the current experimental condition. Ss were given a 5-min rest period after the fourth block of experimental trials, and remaining blocks were separated by $2 \mathrm{~min}$. Ss were instructed to respond as quickly as possible while maintaining a low error rate. Previous data from these Ss indicated an error rate less than $5 \%$ in these tasks, so no formal analysis of errors is included in the present report.

\section{RESULTS}

Each of the four panels in Fig. 3 contains representative AEPs obtained from one of the four Ss. These averages are based on 60 trials (the first 30 trials in each block) digitized at a rate of $8 \mathrm{msec}$ per point. The epoch begins $550 \mathrm{msec}$ before the onset of $\mathbf{S} 2$ and extends for 950 msec beyond S2. Positivity is indicated by a downward deflection. The two panels on the left are from the occiput (Ss J.C. and A.W.) and those on the right are from the vertex (Ss P.J. and P.T.). All records are from the I conditions, with the solid lines denoting records from the $\mathrm{R}$ series and the dotted lines indicating those from the A series. S1 and S2 onset times are indicated by the outline arrows and solid vertical lines, respectively. Records from the $\mathrm{C}$ conditions were nearly identical to those shown here, except for slight differences in the latency and shape of P300 at the more lengthy SOAs, as considered below.

Despite a certain variability among and within the four Ss, several pertinent features are readily apparent in these and in all remaining records. These features are entirely compatible with, and lend credence to, the hypothesis that $\mathrm{P} 300$ reflects the process of decision making.

(1) P300 is substantially enhanced in the R series, relative to the amplitude of the $A$ series potential at a comparable latency. This amplitude enhancement is apparent in all comparisons between the $\mathrm{R}$ and $\mathrm{A}$ series: for all Ss, SOAs, recording sites, and in both $I$ and $C$ conditions. Moreover, the remaining components of the AEP, both those associated with S1 and those with S2, are little affected by the $R$ vs $A$ manipulation. In contrast to $\mathrm{P} 300$, these components display no sizable or 
Fig. 4. Reaction times for the AI, AC, RI, and $R C$ conditions (bottom panel) and peak latency of $P 300$ in the $R I$ and $R C$ conditions (top panel) as a function of stimulus onset asynchrony, all values being measured from the onset of S2. These functions are averaged across the four $\mathrm{Ss}$.

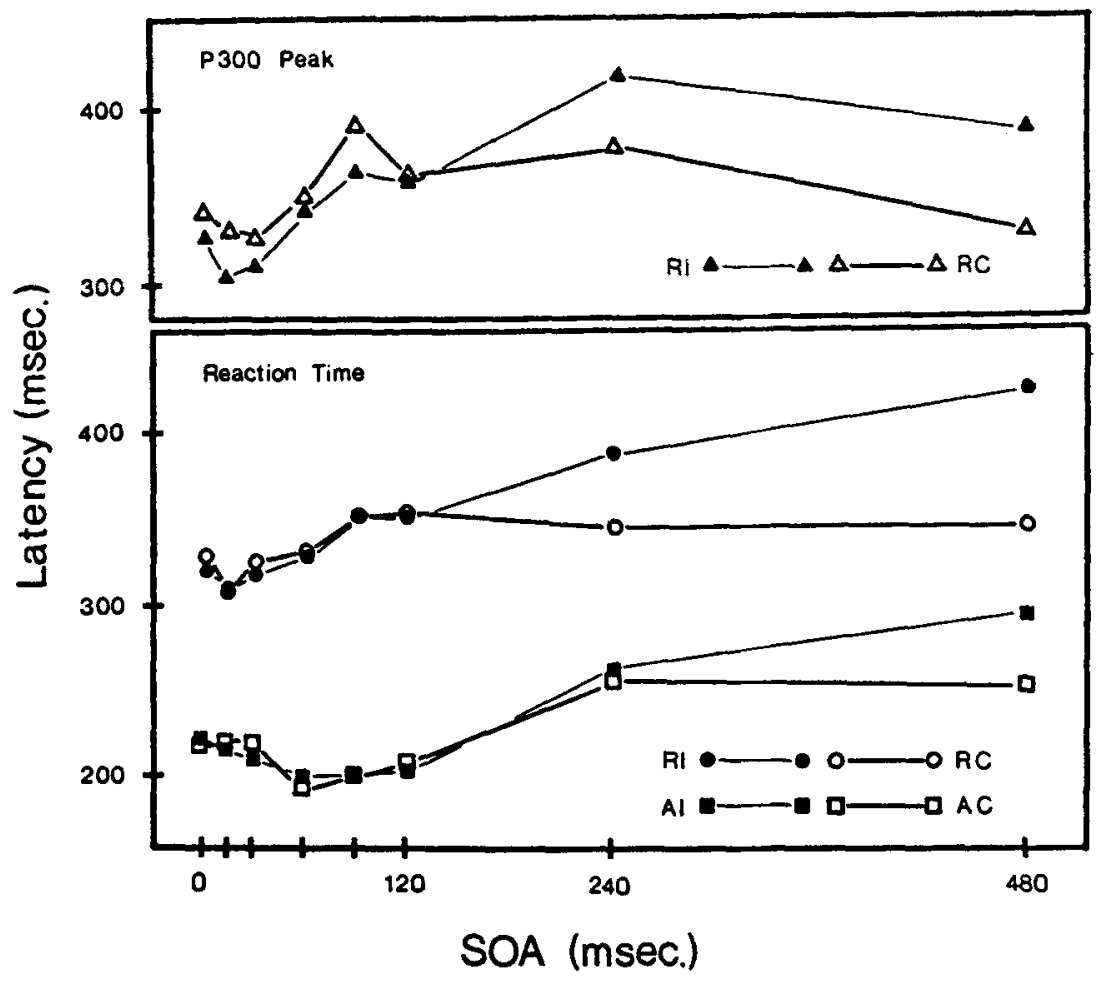

consistent amplitude differences between the two series.

(2) P300 enhancement in the R series is confined to the P300 associated with S2. In contrast, the P300 associated with $S 1$, which can be identified clearly only at the lengthiest SOA, does not approach the amplitude of that associated with $\mathbf{S 2}$, nor is it reliably enhanced in the $R$ series. For only one S (J.C.) was there any enhancement of P300 following S1, and this only at SOA $=480 \mathrm{msec}$ with only a small portion of the enhancement observed following S2 in that condition. The association of $\mathrm{P} 300$ with $\mathrm{S} 2$ rather than with $\mathrm{S} 1$ is confirmed by data presented in the top panel of Fig. 4, in which the peak latency of $\mathrm{P} 300$ following S2 in the R series is plotted as a function of SOA. These data were computed from the vertex records, with the peak latency determined from the printed output of the digital values. P300 was identified as that component which was consistently enhanced during the $R$ series, permitting an unambiguous determination of peak latency in most cases. In the few exceptions where P300 appeared bifurcated or otherwise ill-defined (namely, the records for two Ss at $\mathrm{SOA}=120$ and $240 \mathrm{msec}$ ), latency was measured to the single most positive deflection within the epoch $250-700 \mathrm{msec}$ following S2. The bifurcation in these exceptions appears to be due to an unfavorable compounding of $\mathrm{P} 300$ with late activity (perhaps a small P300) associated with S1.

The latency of $\mathrm{P} 300$ remains fairly stable across all SOAs, and those inflections which are present bear a close resemblance to those in the choice RT functions (plotted in the bottom panel and identified by the circular points). The most conspicuous exception to this close relationship between choice RT and peak P300 latency in the $R$ series occurs at $S O A=480 \mathrm{msec}$. A partial account for this discrepancy may lie in progressive changes in the shape of $\mathrm{P} 300$ at lengthy SOAs. While peak latency shifts as plotted in Fig. 4, P300 also shows progressive amplitude decreases at the longest SOAs and the waveform becomes increasingly skewed toward longer latencies. We attribute these changes in the waveform of $\mathrm{P} 300$ to increased trial-by-trial variability in the latency of P300 at the most lengthy SOAs. Indeed, standard deviations for the choice RTs in the RI condition increased from an average of $80 \mathrm{msec}$ at $\mathrm{SOA}=0 \mathrm{msec}$ to over $110 \mathrm{msec}$ at SOA $=480 \mathrm{msec}$. Comparable increases in the variability of P300 latency would introduce excessive jitter during averaging, which would tend both to depress the amplitude and increase the breadth of $\mathrm{P} 300$ at the longer SOAs. This interpretation is compatible also with data from the longer SOAs in the RC conditions, during which P300 was narrowed and of shorter latency and in which RTs were commensurately shorter and less variable (as would be expected since the continuous presence of $\mathrm{Sl}$ permitted visual integration over all SOAs). Moreover, this interpretation is consistent with previous reports that P300 latency and morphology correlates closely with RT latency and variability (Picton et al, in press; Ritter et al, 1972).

Due to the small amplitudes of P300 in the A series, we were unable to determine reliable peak latencies for P300 in these conditions. The shorter RTs in the A series (identified by the square data points in the bottom panel of Fig. 4) are commensurate with the familiar 
differences between simple and choice RT. There are certain difficulties, however, in interpreting the A series RT functions. Because we used a constant foreperiod and a constant SOA within each session, Ss could well have generated anticipatory responses to S2. Indeed, inspection of trial-by-trial RT scores reveals occasional RT values which are implausibly short. Even at $\mathrm{SOA}=$ $0 \mathrm{msec}$, the constant foreperiod likely encouraged some form of interval estimation.

(3) The association of P300 with S2 differs markedly from the early components which, in contrast, are associated primarily with S1. At all but the longest SOAs, in fact, there are no discernible early components associated with $S 2$, indicating virtually complete refractoriness for these components at the moment of S2 presentation. In subsequently collected data we have observed some refractoriness extending up to SOAs of nearly $1 \mathrm{sec}$.

Whatever the reasons for this unusually protracted recovery function evidenced in the early components, these recovery data have clear implications for the interpretation of P300. The appearance of P300 under conditions in which earlier components are entirely refractory reveals a considerable degree of independence between $\mathrm{P} 300$ and these earlier components.

\section{DISCUSSION}

In the present study, it was determined that P300 enhancement is obtained during tasks requiring a choice $\mathrm{RT}$ (the $\mathrm{R}$ conditions) and that it is enhanced only following the stimulus (S2) permitting this choice and under conditions in which earlier AEP components to that stimulus are partially or wholly absent. These data are consistent with the hypothesis that $\mathrm{P} 300$ reflects a poststimulus process rather than the influences of prestimulus conditions.

It is apparent that the enhancement of $\mathrm{P} 300$ cannot be directly attributed to preparatory fluctuations in attention, either nonspecific or selective in nature. Only S2 elicited a prominent and enhanced P300 in the R series, even though both $\mathbf{S} 1$ and $\mathbf{S} 2$ were presented in close temporal proximity. While it is likely that a heightened state of arousal developed between the warning stimulus and the stripe complex, it is doubtful that any changes could be effected in this state during the short intervals separating S1 and S2. An hypothesis linking P300 amplitude directly to arousal level could be salvaged only by postulating an implausibly rapid shift in arousal between $\mathrm{S} 1$ and $\mathrm{S} 2$.

It also is apparent from the physical configuration of the stimuli that no kind of selective tuning could account for the selective P300 enhancement. It is unlikely that any sort of filter could be tuned both to accept and to reject identical stimulus matrices, nor would filtering seem a desirable strategy in view of the demands of the integration task.

Several prior studies have found selective P300 enhancement in paradigms requiring Ss to observe or to respond selectively only to relevant stimuli. While some of these studies (e.g., Eason et al, 1969) have employed tasks where selective preparation for relevant stimuli could conceivably be effected, others (Donchin \& Cohen, 1967; Harter \& Salmon, 1972) have employed tasks in which relevant and irrelevant stimuli were overlapped in the visual field and were presented in unpredictable sequence. As the latter investigators have speculated, the operation of selectivity in these cases would seem to rely upon principles different from the tuning sort envisaged by Hernandez Peón et al (1956) and others, whereby selectivity follows from the selective modulation of sensory input. Since it is unlikely that sufficiently refined levels of analysis could transpire along afferent pathways, selectivity (and the attendent enhancement of $\mathrm{P} 300$ to relevant stimuli) must surely follow instead from more exhaustive processing of information carried by relevant than by irrelevant stimuli. The results from the above cited experiments concur with the present data, then, in suggesting that $\mathrm{P} 300$ reflects a process invoked by stimulation and that selective tuning is unnecessary and insufficient for P300 enhancement (Hillyard et al, 1973, have drawn similar conclusions from an experiment using auditory stimulation).

Our finding that P300 associated with S1 is not reliably enhanced in the $R$ series indicates also that neither relevance nor information per se determines the amplitude of P300. Our stimuli were so structured that both S1 and S2 delivered information which was relevant and crucial for the judgment required in the $\mathrm{R}$ series. An interpretation of P300 stressing task relevance or information content should predict, we think, that P300 would be enhanced following S1 also. This clearly is not the case. It is only when $S$ is in a position to form his judgment that an enhanced P300 is observed.

The long latency of P300 and its independence from earlier, more sensory-specific components would indicate that P300 is associated with some terminal aspect of S's judgment, by which time sensory properties had been extracted and analyzed (Smith et al, 1970). Since P300 was most prominent in the choice RT tasks and occurred at a latency approximating the choice response latency, we believe this terminal process to be the decision regarding the stimulus. Although the narrow range over which RTs and P300 latencies varied in the present study precluded a reliable determination of the correlation between the two latency measures, our interpretation is in line with data reported by Ritter et al (1972), establishing such a correlation. In vigilance tasks of varying difficulty, P300 latency was observed to vary concurrently with RT over a range in excess of $150 \mathrm{msec}$.

A complicating factor in this relationship is the relative timing of $\mathrm{P} 300$ and the motor response. In both Ritter et al's (1972) study and in many conditions of the present study, the response occurred before the peak of P300. Ritter et al have noted that the actual response marker is preceded by about $45 \mathrm{msec}$ between the 
beginning of the cortical motor protential and EMG activity, plus an additional delay between the initial EMG burst and response execution. The resultant discrepancy between the time of response initiation and the P300 peak would seem to relegate P300 to an inconsequential role in the chain of events determining response latency. Klinke et al (1968), however, have suggested that P300 is part of a complex which includes initial small positive and negative deflections. This suggestion has been confirmed and amplified by Picton et al (in press) and by Ritter et al (1972), who have concluded that the entire complex, and possibly even the onset of P300, begins early enough to be causally implicated in response latency.

It should also be considered that $\mathrm{P} 300$ may reflect the termination of some preparatory state subsequent to the judgment and response, rather than any aspect of the actual decision process (Karlin, 1970). Such an interpretation could account for the differences in P300 following $S 1$ and $S 2$ in the $R$ series, since the preparatory state presumably would terminate only after the response was executed. This interpretation could account also for the differences between the $R$ and $A$ series, if predicated upon the assumption that the preparatory state was greater in the $R$ than in the $A$ series. While this interpretation is entirely compatible with the present data, its applicability is questioned by previous reports that the amplitude of P300 does not strictly depend upon the precursing state of preparation (Donchin et al, 1972; Tueting \& Sutton, 1973). These reports would indicate rather that the amplitude of P300 depends upon the specific process or processes required for successful task resolution.

Although we have interpreted P300 in terms of decision making, it is clear that the term "decision" must be construed loosely. Occasionally we observed P300s (though admittedly of small amplitude) following $\mathrm{S1}$, and in the A series for which no choice decision was required. Donchin et al (1973) have recorded a large P300 in a predictable series under conditions requiring accurate and speedy responding. An alternative term, proposed elsewhere (e.g., Sutton et al, 1967), is the process of "uncertainty resolution," in which case the P300 in these instances could be ascribed to a resolution of temporal uncertainty. In part, we have adopted instead the term "decision" because it does not imply a strict identity with formal information theory. Tueting et al (1971) have reported an imperfect correspondence between information units and P300 amplitude. When their Ss were required to venture a guess concerning an upcoming stimulus, P300 amplitude was monotonically related to the sequential probability of the eliciting stimulus. They also showed, however, that P300 amplitude varied with frequency of occurrence even when Ss were informed beforehand what stimulus would be presented, in which case the same amount of formal information was transmitted by all stimuli.

The shortcomings of formal information units undoubtedly lie in an inability to account for idiosyncratic aspects of S's experience, personal beliefs about the testing situation, and the like, which are at least as important as probabilistic features in determining the significance of stimuli. The results reported here emphasize that it is not the properties of the stimulus as an information carrier, but rather S's activities as an information processor which determine the amplitude of P300. Until a suitable metric is developed for specifying these psychological aspects of information, the term "decision" may serve as a useful descriptor.

\section{REFERENCES}

Donchin, E., \& Cohen, L. Averaged evoked potentials and intramodality selective attention. Electroencephalography \& Clinical Neurophysiology, 1967, 22, 537-546.

Donchin, E., Gerbrandt. L., Leifer, L., \& Tucker, L. Is the contingent negative variation contingent on a motor response? Psychophysiology, 1972, 9, 178-188.

Donchin. E., Kubovy, M., Kutas, M., Johnson, R., \& Herning, R. Graded changes in evoked responses (P300) amplitude as a function of cognitive activity. Perception \& Psychophysies, $1973,14,319-324$.

Eason, R. G., Harter, M. R., \& White, C. T. Effects of attention and arousal on visually evoled cortical potentials and reaction time in man. Physiology \& Behavior, 1969, 4, 283-289.

Eriksen, C. W., Schurman, D. L., \& Richter, O. N-channel tachistoscopes. Behavior Research Methods \& Instrumentation, 1969, 1, 119-122.

Harter, M. R., \& Salmon, L. E. Intram odality selective attention and evoked potentials to randomly presented patterns. Electroencephalography \& Clinical Neurophysiology, 1972,

32, 605-613.
Hermandez Peb́, R., Scherrer, H., \& Jouvet, M. Modification of electrical activity in the cochlear nucleus during attention in unanesthetized cats. Science, 1956, 123, 331-332.

Hillyard, S., Hink, R., Schwent, V., \& Picton, T. Electrical signs of selective attention in the human brain. Science, 1973, 182, 177-180.

Karlin, L. Cognition, preparation and sensory evoked potentials. Psychological Bulletin, 1970, 73, 122-136.

Klinke, R., Fruhstorfer, H., \& Finkenzeller, P. Evoked responses as a function of external and stored information. Electroencephalography \& Clinical Neurophysiology, 1968, 25, 119-122.

Naatanen, R. Evoked potential, EEG, and slow potential correlates of selective attention. Acta Psychologica, 1970,33, 178-192.

Picton, T., Hillyard, S., \& Galambos, R. Cortical evoked responses to omitted stimuli. In M. N. Livanov (Ed.), Major problems of brain electrophysiology. USSR Academy of Sciences, in press.

Ritter, W., Simpson, P., \& Vaughan, H. Association cortex potentials and reaction time in auditory discrimination. Electroencephalography \& Clinical Neurophysiology, 1972, 33, 547-555.

Ritter, W.. Vaughan. H., \& Costa, L. Orienting and habituation to auditory stimuli-a study of short term changes in average evoked responses. Electroencephalography \& Clinical Neurophysiology, $1968,25,550-556$.

Smith, D., Donchin, E., Cohen, L., \& Starr, A. Auditory evoked potentials in man during selective binaural listening. Electroencephalography \& Clinical Neurophysiology, 1970, 28, 146-152.

Sutton, S., Tueting. P., Zubin, J., \& John, E. Evoked potential correlates of stimulus uncertainty. Science, 1965, 150 , 1187-1188.

Sutton, S., Tueting, P., \& Zubin, J. Information delivery and the sensory evoked potentials. Science, 1967, 155, 1436-1439.

Tueting, P., \& Sutton, S. The relationship between prestimulus negative shifts and poststimulus components of the averaged evoked potentials. In S. Kornblum (Ed.), Attention and performance IV. New York: Academic Press, 1973. Pp. 185-207.

Tueting, P., Sutton, S., \& Zubin, J. Quantitative evoked potential correlates of the probability of events. Psychophysiology, 1971, 7, 385-394.

(Received for publication October 15, 1973; revision received December $3,1973$. 